\title{
Improving photo-Fenton process by hydrogen peroxide dosage strategies. Dissolved oxygen performance indicator
}

\author{
Yu Xiangwei ${ }^{a}$, Moisès Graells ${ }^{a}$, Sara Miralles-Cuevas ${ }^{b}$. Alejandro Cabrera-Reina ${ }^{c}$, Montserrat Pérez-Moya ${ }^{a *}$ \\ ${ }^{a}$ Universitat Politècnica de Catalunya, Av. Eduard Maristany, 16, 08019 - Barcelona, Spain \\ ${ }^{b}$ University of Tarapacá, Engineering Faculty, Avda. General Velásquez 1775, Arica, Chile; \\ ${ }^{c}$ University of Almería, Chemical Engineering Dep, Ctra. Sacramento s/n, Almería, Spain; \\ *email. montserrat.perez-moya@upc.edu
}

\begin{abstract}
Fenton and photo-Fenton are Advanced Oxidation processes driven by the supply of hydrogen peroxide $\left(\mathrm{H}_{2} \mathrm{O}_{2}\right)$. $\mathrm{However}$, excessive hydrogen peroxide supply results in unproductive reactions and causes the process performance to decay. Thus, the goal of this work is to systematically compare different $\mathrm{H}_{2} \mathrm{O}_{2}$ dosage strategies using a new approach based on monitoring dissolved oxygen as a performance indicator, which is assumed to directly reveal the inefficient consumption of $\mathrm{H}_{2} \mathrm{O}_{2}$. The systematic approach proposed is a preliminary test to stablish a general methodology to define an optimal dosage scheme.
\end{abstract}

Keywords: Photo-Fenton process, hydrogen peroxide dosage, dissolved oxygen indicator

\section{Introduction}

$\mathrm{H}_{2} \mathrm{O}_{2}$ supply is inherent to Fenton and photo-Fenton processes, since it is the source of the highly oxidant hydroxyl radicals. However, an excessive hydrogen peroxide supply favours reactions consuming such radicals, which is an adverse effect. A product of such reactions is oxygen, and dissolved oxygen concentration (DO) is an interesting measure that can be used as an indirect estimation of the extent of this effect. Thus, the determination of the $\mathrm{H}_{2} \mathrm{O}_{2}$ dosage scheme minimizing such scavenging reactions and maximizing process efficiency is a crucial issue to be addressed.

Supplying the total amount of hydrogen peroxide in convenient portions along the reaction time to improve process performance is reported elsewhere. However, the determination of the best partition as well as the addition timing is not resolved. To address dosage in a systematic way, a pre-established $\mathrm{H}_{2} \mathrm{O}_{2}$ dosage protocol was proposed to improve the performance of the photo-Fenton process (Yamal-Turbay et al. 2012). Although such dosage protocol can be successfully adjusted, it is limited to an initial addition and an initial dosing time, and only two degrees of freedom cannot render enough operation flexibility.

On the other hand, the adaptive addition of $\mathrm{H}_{2} \mathrm{O}_{2}$ depending on monitored process variables, such as DO, has been also investigated (Prieto-Rodríguez et al. 2011; Ortega-Gomez et al. 2012) to overcome the limitations of a pre-fixed scheme. However, lacking of a reliable model, such approach heavily relies on the underlying hypothesis of a convenient DO set-point.

In order to produce a deeper understanding of the relationship between dosage and DO, a research work is proposed to systematically compare different $\mathrm{H}_{2} \mathrm{O}_{2}$ dosage strategies based on dissolved oxygen as performance indicator (assuming a direct relation with the inefficient consumption of $\mathrm{H}_{2} \mathrm{O}_{2}$ ). The approach conceived consists in dividing the reaction span in three stages and addressing them separately, assuming their different qualitative effects in the reaction outcome.

A first effect (initial stage) is given by the initial $\mathrm{H}_{2} \mathrm{O}_{2}$ addition (the reaction kick-off, $\mathrm{A}_{0}$ ); this was studied using form $20 \%$ to twice the stoichiometric amount of $\mathrm{H}_{2} \mathrm{O}_{2}, \mathrm{~S}$ (from $0.2 \mathrm{~S}$ to $2 \mathrm{~S}$ ), and measuring the initial mineralization rate $\left(-d[T O C] /\left.d t\right|_{t=0}\right.$ ) as a performance indicator. A second effect (transition stage), is given by a continuous dosage of $\mathrm{H}_{2} \mathrm{O}_{2}$ aimed at keeping the previous mineralization rate; for a fixed $\mathrm{H}_{2} \mathrm{O}_{2}$ inlet flow, this was studied by dosing until different DO levels (DOstop) were attained at time $\theta$ and measuring the DO change rate $\left(d[D O] /\left.d t\right|_{t=\theta}\right)$ as a performance indicator. A last effect, during a final automatic control stage, would be given by the dosage of $\mathrm{H}_{2} \mathrm{O}_{2}$ driven by an automatic system tuned to control DO. This last effect strongly depends on the decisions made at the previous stages. Hence, this work addresses the study of the first two stages aimed at discussing the ulterior influence on the automatic dosage. Table 1 summarizes the experimental design for these two stages.

Table 1: Stages of the experimental approach.

\begin{tabular}{|l|l|l|}
\hline Stage & Decision variables & Measured outcome \\
\hline Initial & Initial $\mathrm{H}_{2} \mathrm{O}_{2}$ amount: $0.2 \mathrm{~S} \leq \mathrm{A}_{0} \leq 2 \mathrm{~S}$ & $-d[T O C] /\left.d t\right|_{t=0}$ \\
\hline Transition & Stop level for dosage: $4 \mathrm{mg} / \mathrm{L} \leq$ DOstop $\leq$ to $12 \mathrm{mg} / \mathrm{L}\left(@ \mathrm{H}_{2} \mathrm{O}_{2}\right.$ flow $\left.0.287 \mathrm{~mL} / \mathrm{min}\right)$ & $d[D O] /\left.d t\right|_{t=\theta}$ \\
\hline
\end{tabular}

\section{Materials and methods}

Photo-Fenton experiments were performed in a 15-L pilot plant composed by a glass jacketed reservoir tank and a glass annular photo-reactor, and equipped with an Actinic BL TL-DK 36 W/10 1SL lamp (UVA-UVB). The irradiated volume of the latter is $1.5 \mathrm{~L}$, or rather $10 \%$ of the total volume: $\mathrm{pH}$ was adjusted to 2.8 , hydrogen peroxide was used at $33 \%$ (w/w), and iron sulphate $\left(\mathrm{FeSO}_{4} \cdot 7 \mathrm{H}_{2} \mathrm{O}\right)$ was adopted as the source of ferrous ion $\left(\mathrm{Fe}^{2+}\right)$. Paracetamol dissolved in water $(100 \mathrm{mg} / \mathrm{L})$ with an initial total organic carbon (TOC) concentration equal to $65 \mathrm{mg} / \mathrm{L}$ was selected as model wastewater. Duplicate measurements were performed. Total organic carbon (TOC) concentration was determined with a TOC (TOC-VCSH/CSN Shimadzu; Kyoto, Japan) analyser. PCT concentration was measured via HPLC Agilent 1200 series (Agilent Technologies) and $\mathrm{H}_{2} \mathrm{O}_{2}$ concentration was measured by using the spectrophotometric method. 


\section{Results and discussion}

For the first stage, the results showed that the highest initial mineralization rates $\left(-d[T O C] /\left.d t\right|_{t=0}\right)$ are obtained for concentrations between $0.4 \mathrm{~S}$ and $0.8 \mathrm{~S}$ (Figure 1). Thus, an initial concentration of $0.4 \mathrm{~S}$ was selected for the assays in the second stage because exceeding this concentration produced no improvement on process efficiency, while below $0.4 \mathrm{~S}$ the process was slower (probably due to the lack of radicals).

For the second stage, continuous dosage was fixed at $0.287 \mathrm{~mL} / \mathrm{min}$ according to preliminary assays. This continuous addition is expected to keep the mineralization rate $(d[T O C] / d t)$. Figure $2 \mathrm{~A}$ represents the normalized TOC degradation curves and Figure 2B represents the DO evolution.

A

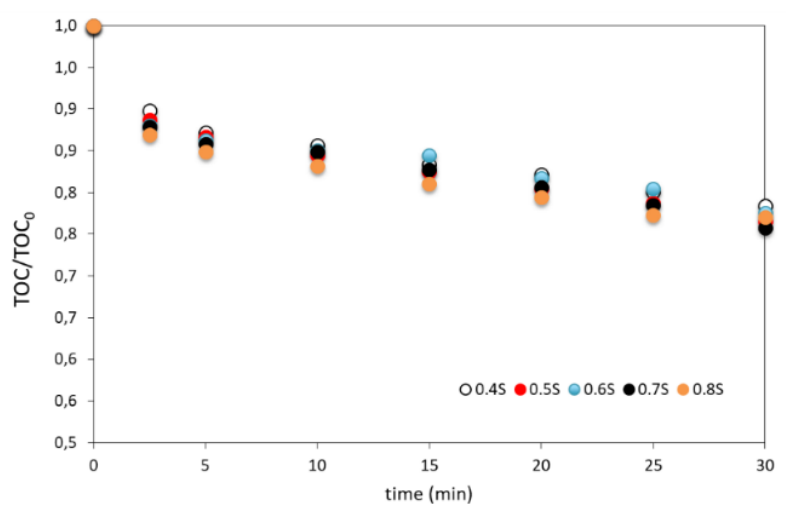

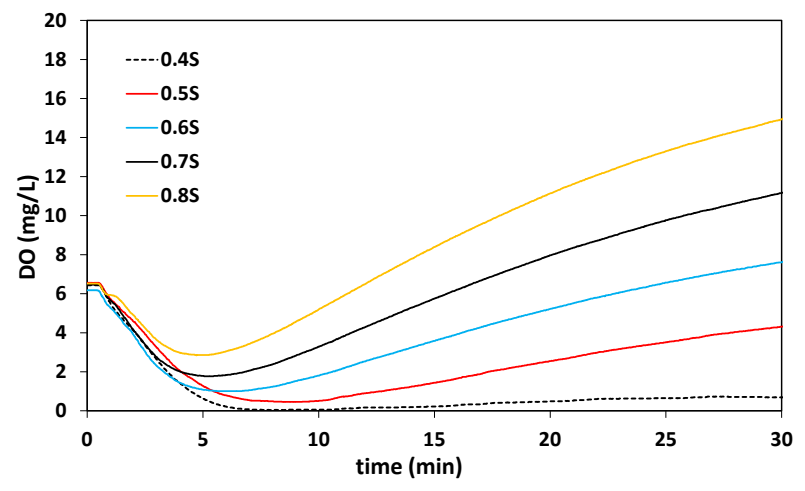

Figure 1: Normalized TOC degradation curves as a function of initial $\mathrm{H}_{2} \mathrm{O}_{2}$ initial dosage

A

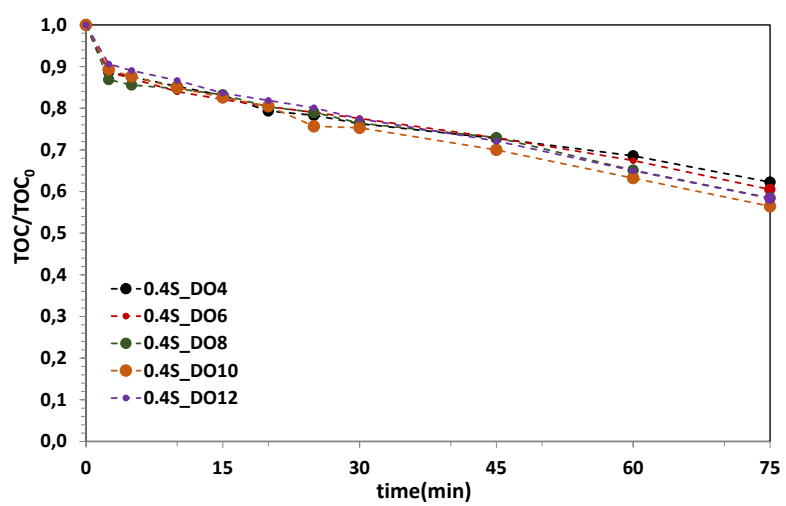

$\mathrm{B}$

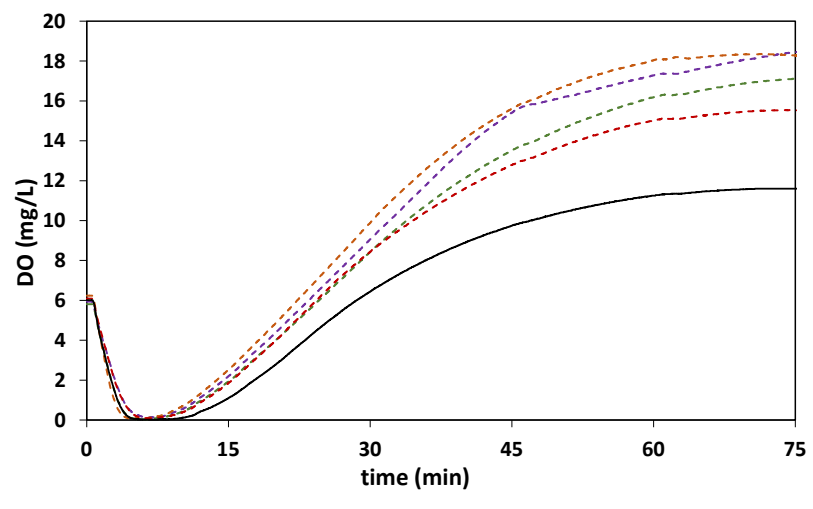

Figure 2: A) Normalized TOC degradation curves as a function of initial $\mathrm{H}_{2} \mathrm{O}_{2}$ initial dosage plus a continuous flow rate in $0.287 \mathrm{~mL} / \mathrm{min}$ that stop addition after specific DOstop value. B) DO evolution

Attaining a DO value of $4 \mathrm{mg} / \mathrm{L}$ (DOstop) seems the most promising result. Longer dosage produces higher $\mathrm{H}_{2} \mathrm{O}_{2}$ concentrations, but this excess is not effectively used because the reaction rate $(-d[T O C] / d t)$ is obviously decreasing and the reagent consumption is rising up. The results obtained were compared in order to determine the best dosage conditions bearing in mind a future subsequent addition of $\mathrm{H}_{2} \mathrm{O}_{2}$ (third control stage) that would, from this point on, automatically determine $\mathrm{H}_{2} \mathrm{O}_{2}$ dosage level (OP) from the measured DO (PV) and a convenient DO value (SP).

\section{Conclusions}

The dosage strategy proposed was implemented and assessed at each stage. Given the quantitative criterion, the quantitative results obtained allowed sorting out the alternatives and identifying the best one. The work contributes in the continue progressing towards improving photo-Fenton process by hydrogen peroxide dosage strategies of photo-Fenton processes.

\section{Acknowledgements}

This work was supported by the Spanish "Ministerio de Economía, Industria y Competitividad (MINECO)" and the European Regional Development Fund, both funding the research Project AIMS (DPI2017-87435-R). Yu Xiangwei particularly acknowledges the State Scholarship Fund of China Scholarship Council (No. 201706950041). A. Cabrera-Reina wants to thank SERC Chile (FONDAP/15110019). Sara Miralles Cuevas wants to thank the Spanish Ministry of Science, Innovation and Universities for her Juan de la Cierva Scholarship.

\section{References}

Ortega-Gomez E, Moreno Ubeda JC, Alvarez Hervas JD, Casas Lopez JL, Santos-Juanes Jorda L, Sanchez Perez JA (2012) Automatic dosage of hydrogen peroxide in solar photo-Fenton plants: development of a control strategy for efficiency enhancement Journal of hazardous materials 237-238:223-230 doi:10.1016/j.jhazmat.2012.08.031

Prieto-Rodríguez L, Oller I, Zapata A, Agüera A, Malato S (2011) Hydrogen peroxide automatic dosing based on dissolved oxygen 
14th Mediterranean Congress of Chemical Engineering - 16-20 November 2020

https://doi.org/10.48158/MeCCE-14.DG.09.15

concentration during solar photo-Fenton Catalysis Today 161:247-254 doi:10.1016/j.cattod.2010.11.017

Yamal-Turbay E, Graells M, Pérez-Moya M (2012) Systematic Assessment of the Influence of Hydrogen Peroxide Dosage on Caffeine Degradation by the Photo-Fenton Process Industrial \& Engineering Chemistry Research 51:4770-4778 doi:10.1021/ie202256k 